\title{
Development of an IEC Neutron Source for NDE
}

\author{
R. A. Anderl*, J. K. Hartwell*, J. H. Nadler", \\ Idaho National Engineering Laboratory \\ *Lockheed Idaho Technologies Company \\ "US Department of Energy, Idaho Operations Office \\ P. O. Box 1625, Idaho Falls ID 83415-7113 \\ J. M. DeMora, R. A. Stubbers and G. H. Miley \\ University of Illinois, Fusion Studies Laboratory \\ 100 NEL, 103 South Goodwin, Urbana IL 61801-2984
}

\begin{abstract}
This paper concerns the development of a neutron source based on the inertial electrostatic confinement (IEC) of a low density fusion plasma in a gridded, spherically-focusing device. With the motivation of using such sources for nondestructive evaluation (NDE) applications, the focus of the development is on : small-size devices, sealed operation with $D_{2}$ or $D_{2} / T_{2}$ mixtures, power-utilization and neutronoutput optimization, and integration into an assay system. In this paper, we describe an experimental system that has been established for the development and testing of IEC neutron sources, and we present preliminary results of tests conducted for $25-\mathrm{cm}$ and $15-\mathrm{cm}$ diameter IEC devices.
\end{abstract}

\section{INTRODUCTION}

Neutron sources are key components in many nondestructive evaluation (NDE) applications. The basis of their use is as follows. Energetic neutrons penetrate deeply into materials and produce excited nuclei via neutron capture and inelastic scattering interactions with atomic nuclei. As the nuclei deexcite, gamma radiation is emitted that is characteristic of the elemental species. Measurement of the gamma-radiation provides a means to identify and quantify the elemental composition of the material [1]. Some NDE applications of this technique are: detection of explosives and chemical agents [2,3], chemical identification in waste-form materials prior to or following disposal [4], quantification of sulfur content in coal [1], assay of fissile- and fertile- content in materials [5], and neutron well logging [1].

Different types of neutron sources have been used in prior NDE applications. Radioisotopic sources have found a niche in portable or semi-portable chemical assay systems like the PINS (Portable Isotopic Neutron Spectroscopy) systems that are used to determine the chemical composition of shells or canisters of chemicals [2]. Typically, ${ }^{252} \mathrm{Cf}$ sources have

FWork supported by US Department of Energy, Office of Nonproliferation and National Security, Office of Research And Development, Idaho Operations Office, under DOE Contract DE-AC07-94ID13223. been used for the PINS systems. Such radioisotopic sources offer certain benefits for these applications: they are available as moderately intense $\left(\sim 10^{7} \mathrm{n} / \mathrm{s}\right)$ point sources, suited for semi-portable applications; they require little maintenance; and they are of a reasonable cost. However, such sources have limitations: they require shielded storage and careful handling, they emit neutrons at a continuous rate, the neutron energy is limited to $2.3 \mathrm{MeV}$, and source strength decays with the radioactive half-life of the ${ }^{252} \mathrm{Cf}$. Sealed-tube accelerator neutron sources have been used in neutron well-logging applications [1] and in systems to quantify the chemical composition of explosives and chemical agents [3]. These sources have several benefits: they can produce $2.45 \mathrm{MeV}$ or $14.1 \mathrm{MeV}$ neutrons from D-D or D-T reactions, respectively, with moderate intensities up to $\sim 10^{6} \mathrm{n} / \mathrm{s}$ for D-D neutrons and $\sim 10^{8} \mathrm{n} / \mathrm{s}$ for D-T neutrons, and they can be operated in pulsed or high duty-cycle modes. However, these sources also have limitations: they are relatively complex and somewhat costly, with operational lifetimes of about 500 hours before head-replacement.

In recent years, there has been a growing interest to apply a neutron source based on the inertial electrostatic confinement (IEC) of a low density fusion plasma. This interest traces back to the pioneering work of Hirsch [6] in which the inertial electrostatic confinement of ionized fusion gases was investigated with a spherically-focusing IEC system. This system was equipped with a gridded spherical cathode, $\sim 11.4 \mathrm{~cm}$ in diameter, and six ion guns that were mounted on ports of a $17.8-\mathrm{cm}$ diameter anode. The ion guns were aligned to the geometric center of the device. For a cathode voltage of $-150 \mathrm{kV}$, Hirsch observed $\sim 3 \times 10^{7} \mathrm{n} / \mathrm{s}$ for D-D operation at $1 \mathrm{mTorr}$ chamber pressure and $\sim 5 \times 10^{9} \mathrm{n} / \mathrm{s}$ for D-T operation at 4 mTorr chamber pressure. Corresponding neutron output rates were $-6 \times 10^{6} \mathrm{n} / \mathrm{s}$ and $-6 \times 10^{8} \mathrm{n} / \mathrm{s}$, respectively for a cathode voltage of $-70 \mathrm{kV}$.

Neutron output of IEC devices has been explored more recently in the Fusion Studies Laboratory at the University of Illinois (UIUC) [7]. In the work of Nadler [8] and Nadler et al. [9], glow-discharge operation was studied in sphericallyfocusing IEC devices with $30-\mathrm{cm}$ and $61-\mathrm{cm}$ diameter test chambers. Three modes of glow discharge operation were 
observed, Star mode, central-spot mode and Halo mode. Maximum neutron outputs were observed for the Star mode in which radially oriented spokes of visible light were observed from the anode to the center of the spherical-grid cathode where the convergence resulted in an intense bright core. Each spoke passed through a grid opening in the cathode, providing evidence of microchannel focusing of ions and electrons generated in the glow-discharge volume. Systematic studies were made to examine the dependence of neutron output on cathode voltage, glow-discharge current, background gas pressure, and grid transparency. Although maximum D-D neutron output observed in this work was $\sim 5 \times 10^{5} \mathrm{n} / \mathrm{s}$ for $-40-\mathrm{kV}$ operation of the small device, the same device has been subsequently operated at $-70 \mathrm{kV}$ to produce $1 \times 10^{6} \mathrm{n} / \mathrm{s}[10]$, with the neutron yield varying linearly with the discharge current.

Subsequent work at UIUC has been directed at increasing neutron output of spherically-focusing IEC devices and on developing alternate geometry IEC devices. A triple-grid configuration, with electron emitters to create a spherical ion-source region near the anode, has been used to achieve moderate neutron output rates for lower-pressure operation [11]. Promising enhancements of neutron output rates have been achieved in pulsed-operational studies of a spherical, single-grid IEC device, for which neutron output rates were observed to scale with the square of the pulsed discharge current [12]. A novel approach to generating neutrons with a gridless, cylindrical IEC device has been demonstrated by Gu et al. [13], who observed $10^{6} \mathrm{n} / \mathrm{s}$ of $D-D$ neutrons for operating conditions of $-60 \mathrm{kV}$ and $20 \mathrm{~mA}$.

Based on the previous research on IEC devices, we summarize the following regarding their potential use for NDE applications. The devices have a demonstrated neutron output that can be used for such applications. In principle, the devices may be simpler, less costly, and have a higher neutron output than present sealed-tube accelerator neutron sources. In addition, the possibility of developing alternategeometry IEC neutron generators offers the possibility of tailoring the neutron source to application-specific requirements. However, effective use of IEC neutron generators for NDE applications requires additional research and development to address some key issues. IEC research devices have been large, hence there is a need to downsize or develop alternate geometries for efficient neutron utilization in NDE applications. Previous IEC research devices were somewhat power inefficient, requiring high power input for moderate neutron output. Recent work on pulsed operation [12] and triple-grid configurations [11] have identified areas of development to enhance neutron output and reduce power input. Finally, little has been done to address the use of tritium in past IEC devices that have typically operated with deuterium in a continuous flowing mode. Highest neutron output will require $D_{2} / T_{2}$ gas mixtures in a sealed IEC device that implements gas-supply and pressure control.
A primary purpose of this paper is to present the results of our development efforts to optimize the performance of IEC devices for NDE applications. The focus of our research is on those issues identified in the previous paragraph. In the following, we describe an experimental system that has been established for this purpose and we present preliminary results of tests conducted for $25-\mathrm{cm}$ and $15-\mathrm{cm}$ diameter spherical IEC devices operated in a glow-discharge mode. We conclude with our recommendations for future research.

\section{EXPERIMENTAL SETUP}

We have established an experimental capability in the Tritium Research Laboratory at the Idaho National Engineering Laboratory (INEL) for test-development of IEC neutron sources operating with $\mathrm{D}_{2}$ or $\mathrm{D}_{2} / \mathrm{T}_{2}$ gases. The principal capability is an experimental setup with the following key features: (1) $25-\mathrm{cm}$ and $15-\mathrm{cm}$ diameter, ultrahigh-vacuum (UHV) spherical test chambers equipped with multiple ports for evacuation, high-voltage feedthrus, gas inlets, viewing windows, diagnostic instrumentation and auxiliary electron emitters; (2) an oil-free turbomolecular pump and diaphragm backing pump for evacuation of the test chambers to $\sim 10^{-9}$ Torr; (3) a quadrupole mass spectrometer for analysis of the gas supply and of the gas composition in an operating IEC device; (4) capacitance manometers for pressure measurements in the IEC chambers during operation; (5) a gas-supply manifold for controlled introduction of high-purity hydrogen, deuterium or helium into either chamber via a precision, copper/sapphire seat metering valve; and (6) a DC power supply capable of delivering up to $75 \mathrm{kV}$ at $33 \mathrm{~mA}$ to the IEC cathode. The UHV chambers are mounted side-by-side on an experiment table, with the common vacuum pumping station and gassupply manifold mounted below. Each test chamber can be operated in a continuous gas-flow mode or in a static mode when an isolation valve is closed.

Measurements of the neutron output from an operating IEC device were made with a "Long Counter" [14] This instrument consisted of three ${ }^{3} \mathrm{He}$ tubes embedded in a polyethylene block that is surrounded on five sides by borated polyethylene. Both the counter assembly and the preampflier associated with the ${ }^{3} \mathrm{He}$ detectors was covered with copper sheet to reduce the influence of arc-generated, RF noise on the counting electronics. The counter is located such that it can be oriented toward either spherical chamber with its front face (side without the borated polyethylene) a distance of 1 meter from the center of each chamber. Calibration of the counter efficiency was achieved with a ${ }^{252} \mathrm{Cf}$ source $\left(3 \times 10^{6} \mathrm{n} / \mathrm{s}\right)$ that was placed at the center of each chamber and at a reference position midway between the chambers. The detector efficiency was determined to be $1.59 \times 10^{-4}$ for the ${ }^{252} \mathrm{Cf}$ source at the reference position. Wall scattering reduced this efficiency value by $10 \%$ and $8 \%$ for the source in the large and small chambers, respectively. 
A unique, shadow-shielded, cathode insulator support was used to position the wire-mesh cathode at the center of each test chamber. Fig. 1 illustrates the cathode configuration for the small IEC device. The spherical-mesh cathode is attached to a stainless-steel extension that is screwed onto the feedthru electrode. The electrode is surrounded by a coaxial, alumina tube that, in turn, is shadowed by a coaxial larger diameter, alumina tube. Cathode diameters were $\sim 1 / 4$ the test chamber diameters for most testing.

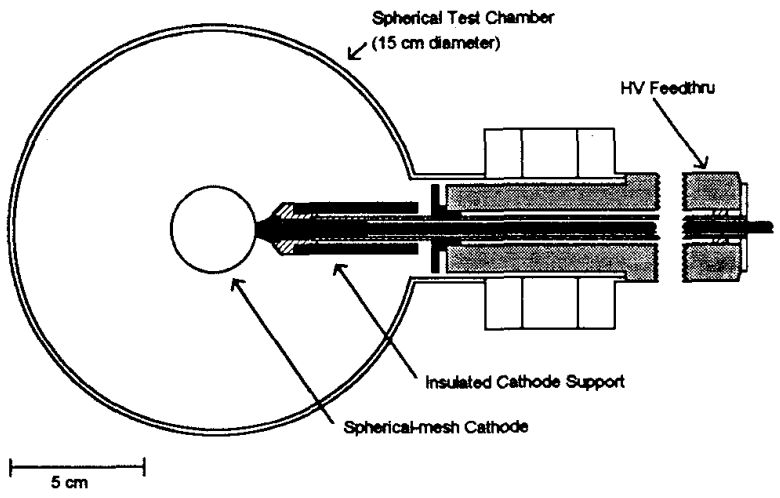

Fig. 1 Insulated cathode support structure for the $15-\mathrm{cm}$ diameter IEC device.

\section{EXPERIMENTAL MEASUREMENTS}

A variety of experimental measurements was made to establish the conditions for operating the $25-\mathrm{cm}$ and $15-\mathrm{cm}$ diameter devices in the Star, glow-discharge mode with both $\mathrm{H}_{2}$ and $\mathrm{D}_{2}$. These included the following: voltage breakdown curves as a function of fill-gas type and pressure, glow-discharge operation of both chambers in a dynamic gas-flow condition and in a sealed condition, and neutron output as a function of input power. Key features of these measurements are presented in the following paragraphs.

Voltage-breakdown curves are given in Fig. 2 for the two INEL IEC devices and for two UIUC spherical IEC devices. Data shown for the INEL devices include measurements with $\mathrm{H}_{2}$ in both chambers and with $\mathrm{D}_{2}$ in the $25-\mathrm{cm}$ diameter chamber. Only $\mathrm{D}_{2}$ gas-breakdown data are shown for the UIUC devices. Breakdown voltages correspond to the voltage values that sustain the glow discharge at the various pressures. As recommended by Nadler [8], the breakdown data are plotted as a function of gas pressure $\mathrm{x}$ device diameter. These results reveal a significant difference between breakdown in $\mathrm{H}_{2}$ versus $\mathrm{D}_{2}$, as observed by Hochberg [15], and they indicate good consistency between the INEL measurements and those by Nadler [8].

Neutron output measurements were made for the $25-\mathrm{cm}$ diameter device operating in the Star, glow-discharge mode at a nominal pressure of $9 \mathrm{mTorr}$ and under continuous $\mathrm{D}_{2}$ gas flow. The results are presented in Fig. 3 as a function of input power, where input power is the product of the operating voltage and the discharge current. For these measurements, the voltage range was $-15 \mathrm{kV}$ to $-50 \mathrm{kV}$. Discharge currents varied from $9 \mathrm{~mA}$ to $20 \mathrm{~mA}$. The data shown in Fig. 3 are consistent with those observed by Nadler [8], and they indicate that a substantial power input is required to achieve high neutron output for a gridded spherical IEC device operating in the glow-discharge mode.

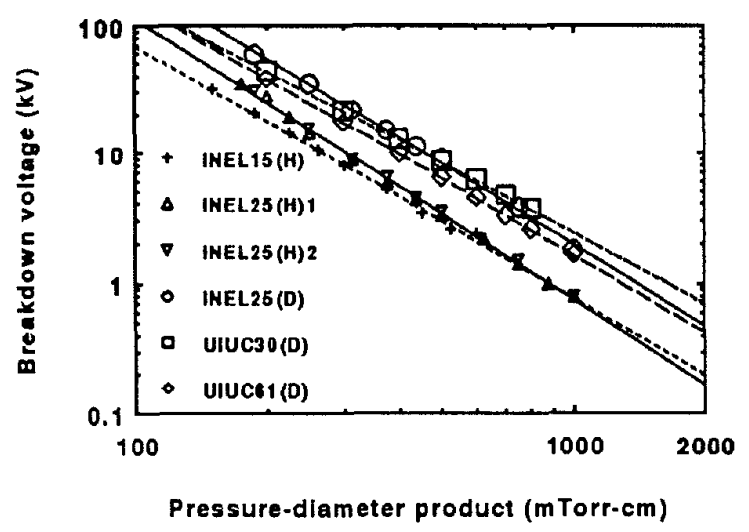

Fig. 2 Comparison of voltage-breakdown curves for spherical IEC devices with different diameters.

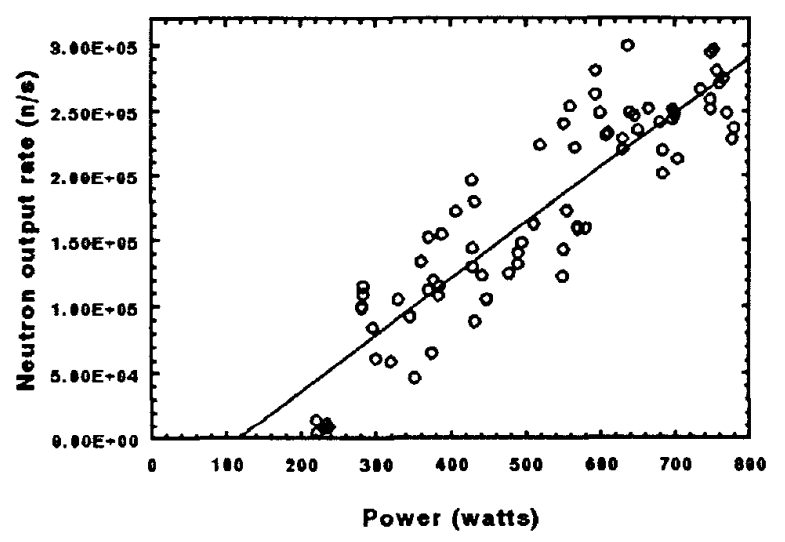

Fig. 3 Output neutron rate for the $25-\mathrm{cm}$ diameter device as a function of input power for Star-mode operation.

Both INEL devices were tested for glow-discharge operation under a static gas-fill (isolation valves were closed). Fig. 4 displays the results of such tests for the $25-\mathrm{cm}$ diameter chamber that was filled with $\mathrm{H}_{2}$ to a pressure of $30 \mathrm{mT}$ Torr, prior to glow-discharge operation at three different starting power level settings, $40 \%, 50 \%$ and $60 \%$. In all cases we observed a systematic reduction in pressure and a consequent rise in cathode voltage, until the applied voltage was terminated. Subsequently, the pressure increased very slowly in the test chamber. The relatively fast drop-off in pressure 
during glow-discharge operation and the subsequent slow pressure rise following discharge termination is indicative of neutral gas pumping to the chamber walls during the glow discharge and slow gas release from the walls afterwards. Neutral-gas pumping occurs when energetic deuterium ions charge-exchange with background gas molecules, resulting in energetic neutral atoms that are no longer constrained by the electric field and are implanted into the interior surface of the chamber. From an analysis of the pressure-decay curves in Fig. 3, we estimated a hydrogen wall-pumping rate of $\sim 10^{-2} \mathrm{~atm}-\mathrm{cm}^{3} / \mathrm{s}$. This pumping speed is comparable to that of a $40 \mathrm{l} / \mathrm{s}$ turbomolecular pump operating at a pressure of 2 mTorr. A similar phenomenon was observed for glowdischarge operation of the $15-\mathrm{cm}$ device. The wall-pumping effect could be compensated for by opening the gas control valve to maintain a constant pressure in the test chamber.

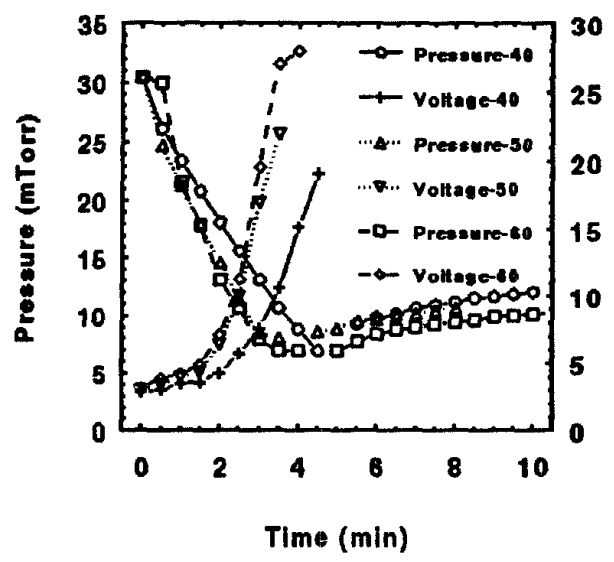

Fig. 4 Pressure and voltage trend data for Star-mode operation of the sealed $25-\mathrm{cm}$ diameter device.

We also observed a pulsating Star-mode operation (visible spokes would cyclically appear and disappear) when the pressure in an IEC device was reduced to a critical value. During this mode, noise was observed from the device. We believe this mode is due to device pressure instability near the threshold for sustained gas breakdown and to the effects of wall-pumping and wall-desorption of hydrogen.

\section{CONCLUSIONS}

To summarize, this paper presented a brief assessment of the potential for using spherically-focusing, IEC devices as neutron sources for NDE applications, and it described an experimental system that has been implemented for performance testing of such sources. Based on the results of this work, we have identified the following research needs for effective implementation of such sources: (1) down-size scaling for effective neutron utilization, (2) development of an auxiliary gas supply and pressure control system for operation of a sealed IEC device with $\mathrm{D}_{2} / \mathrm{T}_{2}$ mixtures, and
(3) grid-design optimization and operation at reduced pressures to achieve higher neutron output and power efficiency. Research is underway to achieve these goals.

\section{REFERENCES}

[1] R. E. Chrien, "Practical uses of neutron-capture gamma-ray spectroscopy," Ch. 7 in Neutron Radiative Capture, R. E. Chrien, editor, Pergamon Press, Oxford, 1984.

[2] A. J. Caffrey, J. D. Cole, R. J. Gehrke and R. C. Greenwood, "Chemical warfare agent and high explosive identification by spectroscopy of neutron-induced gamma Rays," IEEE Trans. on Nucl. Sci., vol. 39, pp 1422-1426, 1992.

[3] R. A. Alvarez, A. D. Dougan, T. F. Wang, and M. S. Rowland, "Neutron interrogation to identify chemical elements with an iontube neutron source (INS)," UCRL-JC-115229, Lawrence Livermore National Laboratory, 1994.

[4] R. J. Gehrke, R. J. Pawelko, and R. C. Greenwood, "Application of PGNAA to preincineration assay of combustible waste for chlorine," Trans. Am. Nucl. Soc., vol. 72, pp 117-118, 1995.

[5] E. Rhodes, C. E. Dickerman and A. DeVolpi, "Radiation interrogation for verification of chemical and nuclear weapons," IEEE Trans. on Nucl. Sci., vol. 39, pp 1041-1045, 1992.

[6] R. L. Hirsch, "Inertial-electrostatic confinement of ionized fusion gases," J. of Appl. Phy. vol. 38, pp 4522-4534, 1967.

[7] G. H. Miley, "Grid physics, IEC simulations, and scaling: U. of Illinois experiments, plans and perspectives," presented at the Physics of Spherical Continuous Inertial Fusion Workshop, Sante Fe, NM, January 11-13, 1995, unpublished.

[8] J. H. Nadler, "Space-charge dynamics and neutron generation in an inertial-electrostatic confinement device," Ph D. Thesis, Nuclear Engineering, University of Illinois, 1992.

[9] J. H. Nadler, G. H. Miley, Y. Gu, and T. Hochberg, "Characterization of an inertial-electrostatic confinement glow discharge (IECGD) neutron generator," Fusion Tech. vol. 21, pp 1639-1643, 1992

[10] G. H. Miley et al., "Inertial-electrostatic confinement neutron/proton source," Third International Conference on Dense Z-Pinches, AIP Conference Proceedings 299, pp 675-689, American Institute of Physics, New York, 1993.

[11] J. B. Javedani, "IEC triple grid," paper presented at the Physics of Spherical Continuous Inertial Fusion Workshop, Sante Fe, NM, January 11-13, 1995, unpublished.

[12] Y. Gu and G. Miley, "Pulsed IEC neutron generator," Proceedings, 10th IEEE Interm. Pulsed Power Conference, 1995, in press.

[13] Y. Gu, J. B. Javedani and G. H. Miley, "A portable cylindrical electrostatic-fusion device for neutronic tomography," Fusion Tech., vol. 26, pp 929-932, 1994.

[14] L.V. East and R. B. Walton, "Polyethylene moderated ${ }^{3} \mathrm{He}$ neutron detectors," Nucl. Instr. Meth. vol. 72, p 161, 1969

[15] T. A. Hochberg, "Characterization and modeling of the gas discharge in a SFID neutron generator," Master's Thesis, Nuclear Engineering, University of Hllinois, 1989. 